\title{
The tissue differentiation and cancer manifolds in gene and protein expression spaces
}

\author{
J Nieves ${ }^{1}$ and A Gonzalez ${ }^{2}$ \\ ${ }^{1}$ Faculty of Physics, University of Havana, Cuba \\ ${ }^{2}$ Institute of Cybernetics, Mathematics and Physics, Havana, Cuba \\ E-mail: joan.nieves@estudiantes.fisica.uh.cu
}

September 2021

\begin{abstract}
It is well known that, for a particular tissue, the homeostatic and cancer attractors are well apart both in gene expression and in protein expression spaces. By using data for 15 tissues and the corresponding tumors from The Cancer Genome Atlas, and for 49 normal tissues and 20 tumors from The Human Protein Atlas, we show that the set of normal attractors are also well separated from the set of tumors. Roughly speaking, one may say that there is a cancer progression axis orthogonal to the normal tissue differentiation and cancer manifolds. This separation suggests that therapies targeting common genes, which define the cancer axis, may be effective, irrespective of the tissue of origin.
\end{abstract}

\section{Introduction}

In gene expression (GE) space, the homeostatic and cancer phenotypes for a given tissue span distant regions allowing an easy classification of samples $[1,2]$. The transition between these two radically different states of the tissue is understood to proceed discontinuously, through the rearrangement of the expressions of thousands of genes [3]. A similar situation takes place in protein expression (PE) space [4].

On the other hand, different tissues in the human body are also well separated in GE or PE spaces. We understand that this separation has a epigenetic origin [5]. The set of all human tissues span a tissue differentiation manifold, which experiences only minor changes during the individual lifespan because of its homeostatic nature.

In the present paper, we show that the set of cancer attractors also span a manifold in GE or PE spaces well apart from the tissue differentiation manifold. As a consequence, we may also define a main direction indicating cancer progression. This direction may slightly differ from the vector connecting the normal and cancer attractors for any specific tissue, and should be more likely related to genes playing an important role in any cancer.

We use RNA-seq GE data for 15 tissues and their corresponding tumors, obtained from The Cancer Genome Atlas portal (TCGA, https://www.cancer.gov/tcga) [6], and 
immuno-histo-chemistry PE data for 49 normal tissues and 20 tumors coming from the Human Protein Atlas (HPA, https://www.proteinatlas.org) [7]. The data is processed by means of a Principal Component Analysis (PCA) technique [8]. 3D plots arising from the first 3 principal components allow the identification of the tissue differentiation and cancer manifolds and an approximate description of the cancer direction.

\section{The tissue differentiation and cancer manifolds in GE space.}

The PCA technique allows a distinct separation between normal samples and tumors, as it can be seen, for example, in Supplementary Figure I for Liver Hepatocellular Carcinoma [2]. Normal and tumor samples are grouped in clouds, the centers of which define the homeostatic and cancer attractors, respectively.

We compute center positions (mean geometric averages of sample clouds) for the 15 tissues and their corresponding tumors, listed in Supplementary Table I, and apply PCA to obtain the three main axes of maximal variance (see Supplementary Table II). Data, Python routines and results may be found in the GitHub repository described below.

The results are presented in figure 1, top panel, where a clear separation between normal and tumor centers is apparent. There is a region or manifold for normal tissues, which may be understood in terms of tissue differentiation, but there is also a region or manifold for tumors. Figure 1 bottom panel draws a schematics for this separation, where manifolds are represented by surfaces and a cancer axis is defined by the orthogonal vector.

It is quite interesting that genes consistently over- or under-expressed in all of these 15 tumors play also an important role in the unitary vectors along the principal component axes [2]. In other words, they play a role both in cancer development and tissue differentiation. This is the case, for example, of the UBE2C gene, which high expression values are related to low cell differentiation and tumors [9, 10], or of ADH1B, a gene participating in the oxidative metabolism of ethanol, which role in cancer is recognized [11], but its participation in basic tissue differentiation is less understood $[12]$.

In order to get at least a rough description of the cancer axis we compute the vector connecting the center of the normal region with the center of the tumor region. The five over-expressed genes with the highest contribution to this vector are the following: MMP11, CST1, UBE2C, MYBL2 and COL10A1. MMP11 and UBE2C are consistently over-expressed in the 15 tissues under study [2]. On the other hand, the five underexpressed (silenced) genes with the highest contribution to the vector are the following: ADH1B, C7, DES, PI16 and DPT. ADH1B and C7 are consistently under-expressed in the set of 15 tissues [2]. In other words, pancancer genes play a crucial role in the main cancer direction. This analysis is limited, involving only a few genes, but illustrative. 

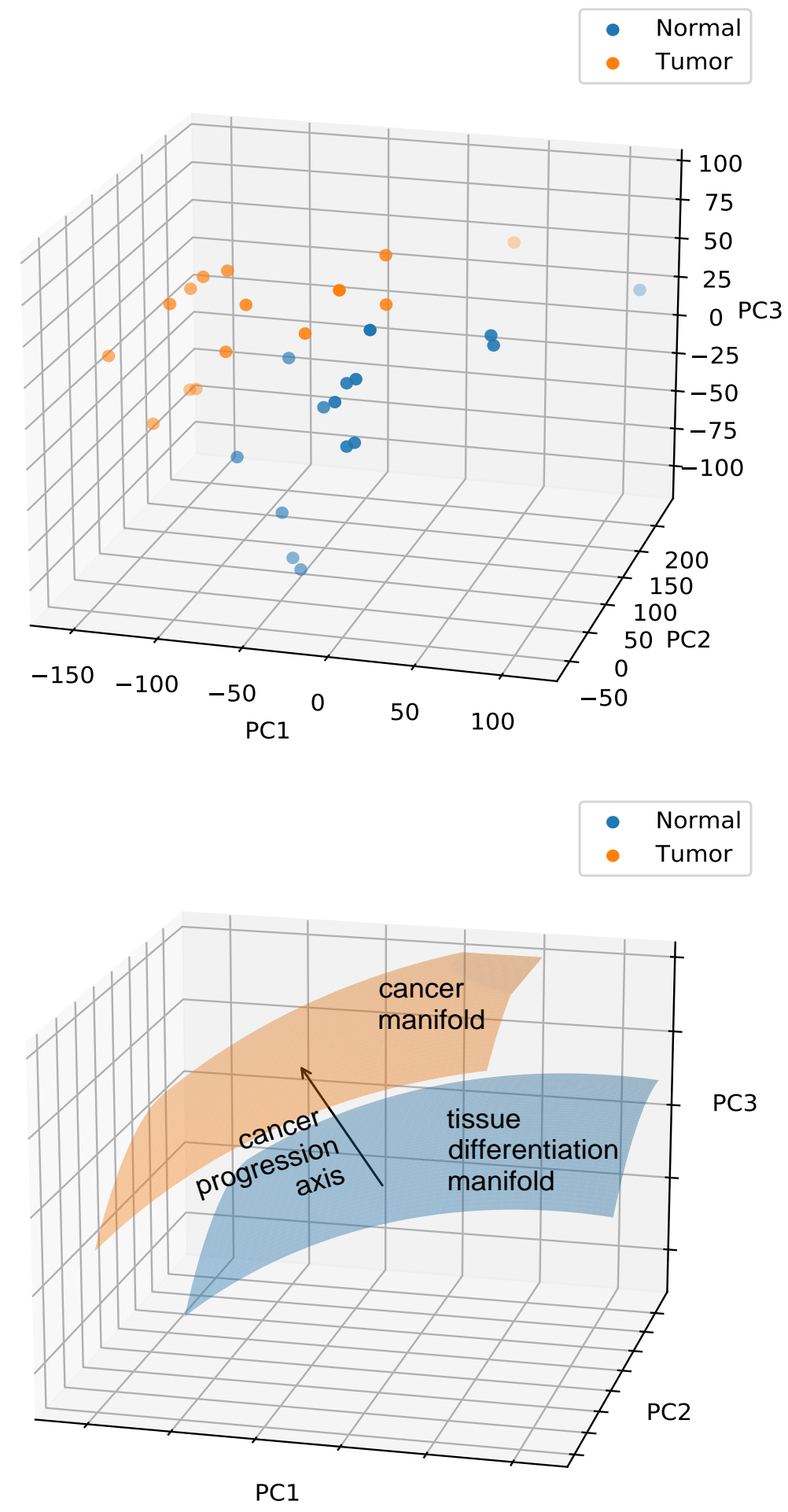

Figure 1: Top: Principal component analysis of the TCGA gene expression data for 15 tissues. The centers of the clouds of samples for each tissue are depicted. Bottom: Schematics of the topology of gene expression space. Normal tissues are distributed along the tissue differentiation manifold whereas cancer attractors are also grouped. There is a cancer progression axis connecting both regions. 


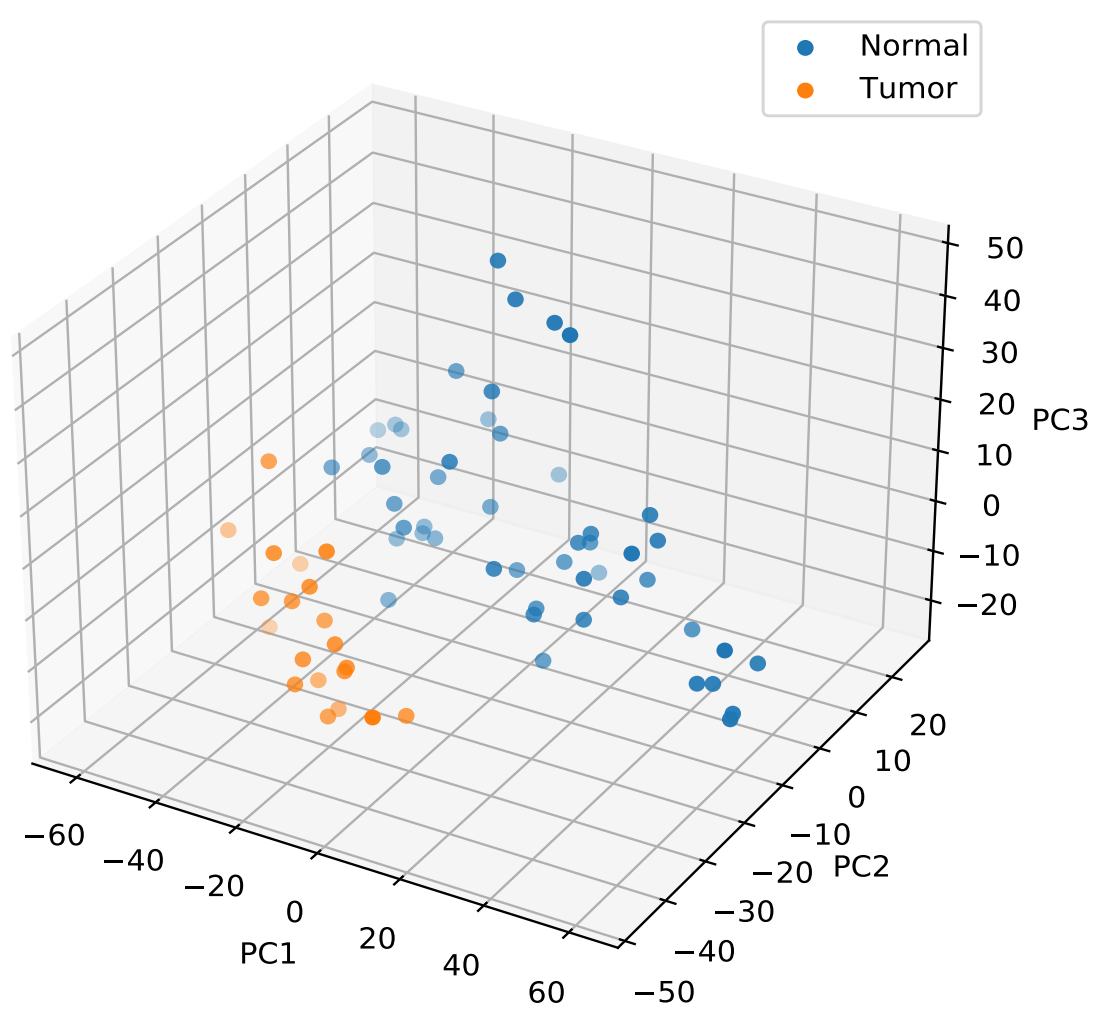

Figure 2: PCA results for the protein expression data of the HPA (49 normal tissues, 20 tumors). As in the previous figure, tissue differentiation and cancer regions are apparent.

\section{The tissue differentiation and cancer manifolds in PE space.}

Protein expression data coming from immuno-histo-chemistry measurements are available in the HPA database. The expression is discretized in four categories: High, Medium, Low and Not Detected. We translate these categories into numerical values in order to apply PCA (see Methods section below). 10166 proteins are consistently expressed in 49 normal tissues and 20 tumors, listed in Supplementary Tables III and IV. The results for the first three principal components are listed in the tables and represented in figure 2 .

The tissue differentiation and cancer manifolds are also apparent in PE space. They are mainly separated along the PC2 direction. The five genes with the most significant amplitudes in the unitary vector along PC2 are: KLC1 (+), ALPK3 (+), DIDO1 (-), CCR9 $(+)$ and GATA4 $(+)$. The notation $(+)$ and () refers to over- and under-expressed genes. The over-expressed genes play known roles in cancer, and the silenced DIDO1 in apoptosis. Other genes, like UBE2C, are well below in the ranking (in the 785th place). We assume that this the difference with regard to the genes coming from the GE data is related to the discrete nature of the PE data, not accounting properly for the magnitude of the differential expression, although it could also be related to regulation of translation. 


\section{Discussion.}

By applying a PCA technique to GE data for 15 normal tissue tumor pairs from the TCGA portal, and to PE data for 49 normal tissues and 20 tumors from the HPA website, we checked that normal tissues span a tissue differentiation manifold, and tumors span a cancer manifold, which are clearly separated one from the other.

Conceptually, one can understand why normal tissues are grouped in GE or PE space. They have a common genetic origin, and differentiation comes from epigenetic events. Pluripotent stem cells undergo a sequence of transformations, leading to tissues, which may be seen as the result of reaching local attractor states of the global regulatory network governing the genoma [13].

With regard to tumors, one expect a cancer attractor related to each one of the homeostatic attractors of the normal tissues of origin [14]. However, there is not an obvious reason for these cancer attractors to be grouped. In our understanding, the best hypothesis to explain this fact is their common evolutionary origin, that is the idea that cancer is an atavistic state of metazoans, leading to modern multicellular organisms $[15]$.

There are other manifestations of cancer universality in spite of its diversity. The known hallmarks, for example $[16,17]$. The Pareto character of the tails in the gene expression distribution functions is also a general characteristic [3], but it is mainly related to complex behaviour [18]. In addition, we checked that the volumes of the basins of attraction for all tumors in GE space are very similar, differently from normal tissues, which exhibit a wider variability [19].

Finally, there is the issue related to the global cancer progression axis and the relevant genes contributing to it. It is hard to imagine a therapy targeting a gene such as UBE2C, which participates in so many important pathways. However, the fact that precisely this kind of genes delineates the border between the normal and cancer manifolds undoubtedly constitute a valuable information, and should have practical consequences in the near future.

\section{Methods}

The method we use to process the TCGA data is described elsewhere [2, 3]. First, we shift the FPKM data by adding a constant 0.1 in such a way that a expression value below 0.1 will never lead to a significant differential expression. Then, cloud centers are computed by geometric averaging over normal and tumor samples, respectively. Each of them represents a point in the 60,483 dimensional abstract gene expression space. As a result, we get 15 points for normal tissues and 15 for their corresponding tumors. Next, we compute a common reference value for each gene, $e_{r e f}$, by geometrically averaging over the normal tissues. Fold variations are defined in logarithmic scale: $e_{f o l d}=\log \left[2, e / e_{r e f}\right]$, such that under- and over-expression values enter the covariance matrix in a symmetrical way. The matrix generated from these 30 points is diagonalized. 
The first three principal components account for 22,16 and $12 \%$ of the total variance, respectively. These 3 components are used to draw figure 1.

With regard to the approximate vector defining the cancer axis, we use the expression $\log \left[2, e_{\text {tumor }} / e_{\text {ref }}\right]$, where $e_{\text {tumor }}$ is the geometric average of the tumor centers.

On the other hand, for the PE analysis we use the normal tissue and pathology data from the HPA. This data is discretized appearing only in four expression categories: High, Medium, Low and Not Detected. We assume that these categories may be translated into three values of the efold variable: $+1,0,-1$, and -1 , respectively. When various lectures are provided for a single tissue we take the average. A total of 10166 proteins are consistently expressed in 49 normal tissues and 20 tumors. This is the dimension of the covariance matrix. The first three principal components emerging after diagonalization account for $41 \%$ of the total variance. They are used to draw figure 2 .

\section{References}

[1] U. Alon, N. Barkai, D. A. Notterman, K. Gish, S. Ybarra, D. Mack, and A. J. Levine. Broad patterns of gene expression revealed by clustering analysis of tumor and normal colon tissues probed by oligonucleotide arrays. Proceedings of the National Academy of Science, 96(12):6745$6750,1999$.

[2] Augusto Gonzalez, Yasser Perera, and Rolando Perez. On the gene expression landscape of cancer. arXiv e-prints, page arXiv:2003.07828, 2020.

[3] Augusto Gonzalez, Joan Nieves, Dario A. Leon, Maria Luisa Bringas Vega, and Pedro Valdes Sosa. Gene expression rearrangements denoting changes in the biological state. Scientific Reports, 11(1):8470, 2021.

[4] Jane M. C. Oh, Franck Brichory, Eric Puravs, Rork Kuick, Chris Wood, Jean Marie Rouillard, John Tra, Sharon Kardia, David Beer, and Samir Hanash. A database of protein expression in lung cancer. PROTEOMICS, 1(10):1303-1319, 2001.

[5] Charles A. Gersbach. Engineered Proteins for Controlling Gene Expression, chapter 13, pages 125-138. Academic Press, second edition, 2013.

[6] Katarzyna Tomczak, Patrycja Czerwiska, and Maciej Wiznerowicz. Review The Cancer Genome Atlas (TCGA): an immeasurable source of knowledge. Contemp Oncol (Pozn), 1A:68-77, 2015.

[7] Mathias Uhlen, Cheng Zhang, Sunjae Lee, Evelina Sjstedt, Linn Fagerberg, Gholamreza Bidkhori, Rui Benfeitas, Muhammad Arif, Zhengtao Liu, Fredrik Edfors, Kemal Sanli, Kalle von Feilitzen, Per Oksvold, Emma Lundberg, Sophia Hober, Peter Nilsson, Johanna Mattsson, Jochen M. Schwenk, Hans Brunnstrm, Bengt Glimelius, Tobias Sjblom, Per-Henrik Edqvist, Dijana Djureinovic, Patrick Micke, Cecilia Lindskog, Adil Mardinoglu, and Fredrik Ponten. A pathology atlas of the human cancer transcriptome. Science, 357(6352):660, 2017.

[8] Jake Lever, Martin Krzywinski, and Naomi Altman. Principal component analysis. Nature Methods, 14(7):641-642, 2017.

[9] Inbal Ben-Eliezer, Yael Pomerantz, Dalia Galiani, Nava Nevo, and Nava Dekel. Appropriate expression of ube2c and ube2s controls the progression of the first meiotic division. The FASEB Journal, 29(11):4670-4681, 2015.

[10] Hassan Dastsooz, Matteo Cereda, Daniela Donna, and Salvatore Oliviero. A comprehensive bioinformatics analysis of UBE2c in cancers. International Journal of Molecular Sciences, 20(9):2228, 2019.

[11] Wojciech Jelski and Maciej Szmitkowski. Alcohol dehydrogenase (ADH) and aldehyde dehydrogenase (ALDH) in the cancer diseases. Clinica Chimica Acta, 395(1-2):1-5, 2008. 
[12] Dvir Netanely. Gene expression analysis of mesenchymal stem cell differentiation and leukemic over expression of tissue specific genes. Master's thesis, Weissmann Institute of Science, Rehovot, Israel, 2006.

[13] Sui Huang, Gabriel Eichler, Yaneer Bar-Yam, and Donald E. Ingber. Cell fates as high-dimensional attractor states of a complex gene regulatory network. Physical Review Letters, 94(12):128701, 2005.

[14] Sui Huang, Ingemar Ernberg, and Stuart Kauffman. Cancer attractors: A systems view of tumors from a gene network dynamics and developmental perspective. Seminars in Cell 83 Developmental Biology, 20(7):869-876, 2009.

[15] P C W Davies and C H Lineweaver. Cancer tumors as metazoa 1.0: tapping genes of ancient ancestors. Physical Biology, 8(1):015001, 2011.

[16] Douglas Hanahan and Robert A Weinberg. The hallmarks of cancer. Cell, 100(1):57-70, 2000.

[17] Douglas Hanahan and Robert A. Weinberg. Hallmarks of cancer: The next generation. Cell, 144(5):646-674, 2011.

[18] Didier Sornette. Probability Distributions in Complex Systems. In Encyclopedia of Complexity and Systems Science. Springer New York, 2009.

[19] Frank Quintela and Augusto Gonzalez. Estimating the number of available states for normal and tumor tissues in gene expression space. arXiv e-prints, page arXiv:2005.02271, 2020.

\section{Acknowledgments}

A.G. acknowledges the Office of External Activities of the Abdus Salam Centre for Theoretical Physics for support. The research is carried on under a project of the Cuban Nuclear Energy and Advanced Technologies Agency (AENTA).

\section{Authors contributions}

A.G. conceived and coordinated the work. J.N. processed GE and PE data and actualized the GitHub repository. Both authors analyzed and interpreted the results, contributed to the manuscript and approved the final version.

\section{Competing interest}

The authors declare that they have no competing interests.

\section{Availability of data and materials}

The information about the data we used, the procedures and results are integrated in a public repository that is part of the project "Processing and Analyzing Mutations and Gene Expression Data in Different Systems": https://github.com/DarioALeonValido/evolp. The TCGA data is replicated in path ../evolp/databases_generated/tcga-hpa/ and the HPA data in ../evolp/databases_external/tcga-hpa/. To process each data set we include specific scripts in ../evolp/tcga-hpa/. The final result of the script for TCGA data is the top panel of figure 1, and the result of script for HPA data is the figure 2. 


\section{Supplementary Information}

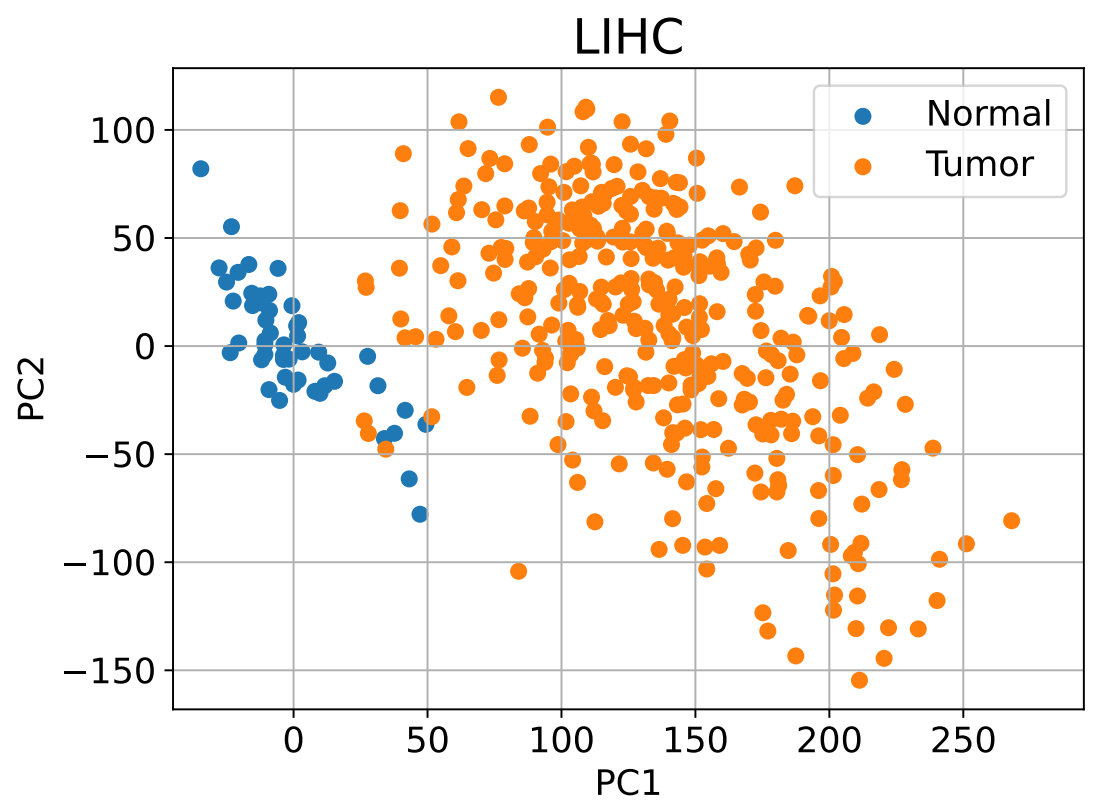

Suppl Figure I: An example taken from Ref. [2]: The (PC1,PC2) plane for the GE data in LIHC (liver hepato-cellular carcinoma). Normal and tumor samples are grouped. Cloud centers define the homeostatic and cancer attractors. The first is at the origin of coordinates by construction. The second is located along the PC1 axis at a distance of around 135 units from the origin [3]. 


\section{UBE2C}
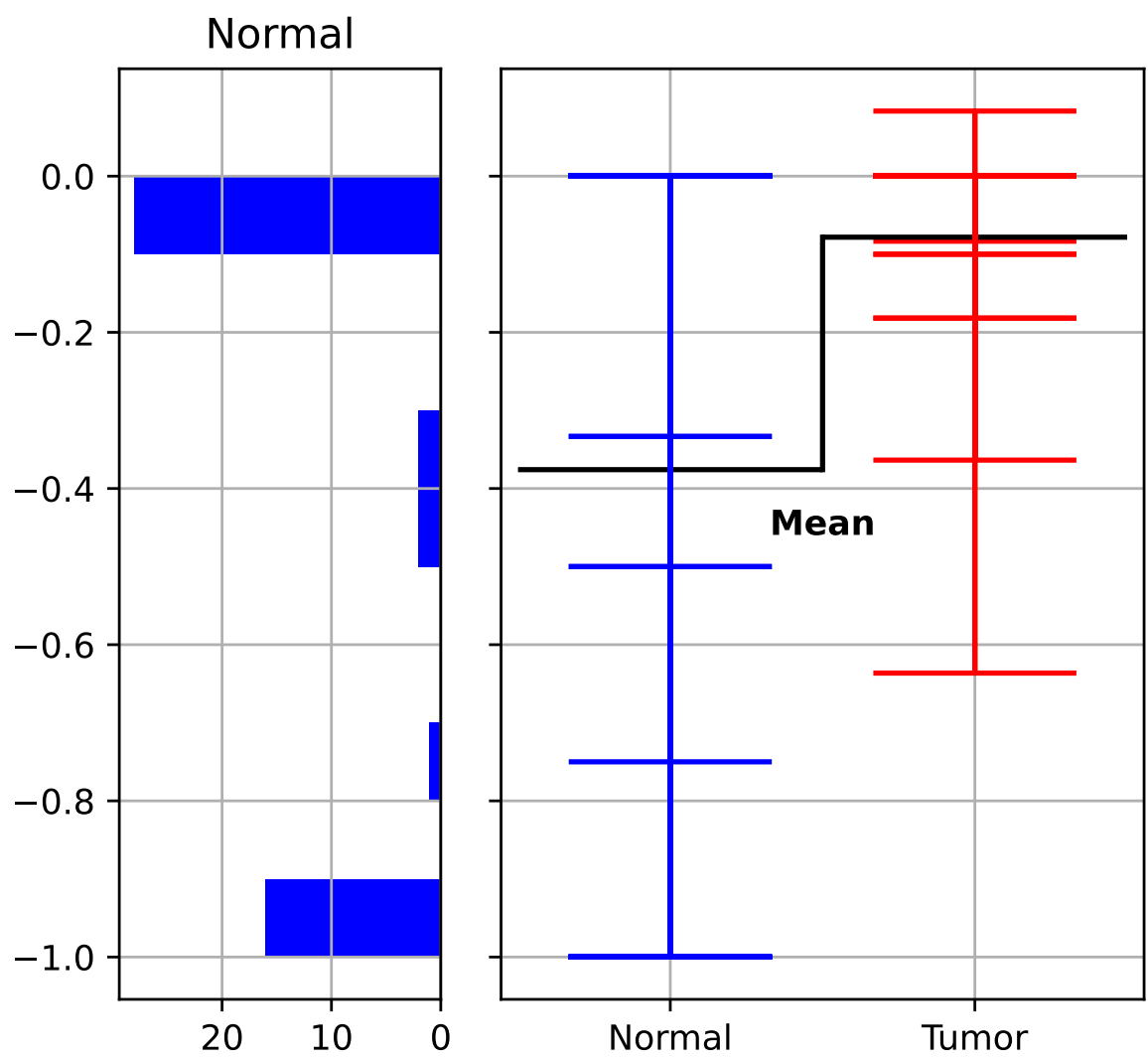

Normal

Tumor

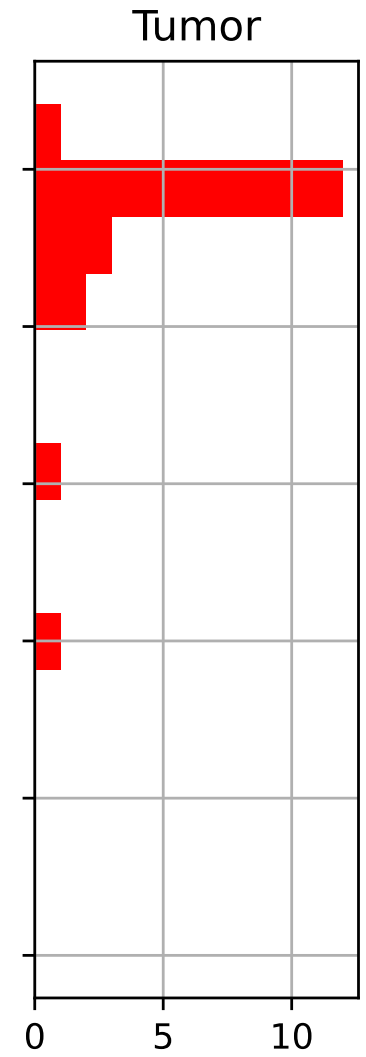

Suppl Figure II: Center: The PE values for the UBE2C protein in different tissues. Mean values, drawn in black, are guides to the eye. The y axis is the fold variation in logarithmic scale. Histograms are drawn in the side panels. The x axes in the histograms correspond to the number of tissues. [3]. 
The tissue differentiation and cancer manifolds

Suppl Table I: The TCGA abbreviations for the 15 tissues under study.

\begin{tabular}{ll}
\hline Abbreviation & Cancer type \\
\hline BLCA & Bladder Urothelial Carcinoma \\
BRCA & Breast invasive carcinoma \\
COAD & Colon adenocarcinoma \\
ESCA & Esophageal carcinoma \\
HNSC & Head and and neck squamous cell carcinoma \\
KIRC & Kidney clear cell carcinoma \\
KIRP & Kidney papillary cell carcinoma \\
LIHC & Liver hepatocellular carcinoma \\
LUAD & Lung adenocarcinoma \\
LUSC & Lung squamous cell carcinoma \\
PRAD & Prostate adenocarcinoma \\
READ & Rectum adenocarcinoma \\
STAD & Stomach adenocarcinoma \\
THCA & Thyroid carcinoma \\
UCEC & Uterine corpus endometrial carcinoma \\
\hline
\end{tabular}

Suppl Table II: Coordinates along the first three PC axes of the centers of normal and tumor sets of samples in GE space for the 15 tissues taken form the TCGA portal. In general, the transition from a normal tissue to its tumor involves displacement along the three coordinates.

\begin{tabular}{lllllll}
\hline & \multicolumn{3}{c}{ Normal } & & \multicolumn{3}{c}{ Tumor } \\
Tissue & & & & & \\
& PC1 & PC2 & PC3 & PC1 & PC2 & PC3 \\
\hline BLCA & -20.51 & -12.76 & 6.70 & -102.25 & 26.85 & 68.06 \\
BRCA & -2.17 & -57.66 & 25.27 & -65.80 & -12.38 & 65.00 \\
COAD & -51.98 & 34.61 & -106.41 & -121.17 & 60.57 & -14.05 \\
ESCA & -77.12 & 5.88 & -35.51 & -156.08 & 13.67 & 17.90 \\
HNSC & -54.21 & 36.47 & 19.26 & -114.12 & 41.14 & 55.71 \\
KIRC & 76.81 & -9.42 & 52.41 & 11.44 & 4.92 & 67.09 \\
KIRP & 74.24 & -4.81 & 56.83 & 7.01 & 21.54 & 92.03 \\
LIHC & 111.81 & 234.51 & 20.71 & 43.73 & 190.23 & 57.54 \\
LUAD & 7.88 & -52.87 & 0.19 & -77.89 & -12.59 & 35.30 \\
LUSC & 3.31 & -52.82 & -2.61 & -115.45 & 3.28 & 56.74 \\
PRAD & 0.04 & -39.94 & 31.52 & -29.06 & -20.65 & 53.23 \\
READ & -42.80 & 18.33 & -107.33 & -118.46 & 64.27 & -14.47 \\
STAD & -52.80 & 14.37 & -70.89 & -131.54 & 19.36 & -24.06 \\
THCA & 19.04 & -61.85 & 71.54 & -5.02 & -36.39 & 85.82 \\
UCEC & 8.45 & -52.04 & 38.35 & -84.06 & 14.20 & 76.80 \\
\hline
\end{tabular}


Suppl Table III: Coordinates along the first three PC axes of the PE values for the 49 normal tissues from the HPA portal.

\begin{tabular}{llllllll}
\hline Tissue & PC1 & PC2 & PC3 & Tissue & PC1 & PC2 & PC3 \\
\hline adipose tissue & -39.06 & 11.70 & -6.88 & oral mucosa & -3.98 & 2.38 & 28.71 \\
adrenal gland & 26.30 & 2.74 & -9.15 & ovary & -31.56 & 3.01 & -3.26 \\
appendix & 3.66 & -9.35 & -0.79 & pancreas & 3.03 & -4.05 & -6.82 \\
bone marrow & -11.64 & 4.59 & 8.04 & parathyroid gland & 6.52 & -3.74 & -16.06 \\
breast & -17.74 & 1.67 & -0.33 & placenta & 13.07 & -0.91 & 4.55 \\
bronchus & 25.54 & -0.37 & 8.23 & prostate & 5.38 & -8.34 & -5.60 \\
caudate & -25.93 & 4.11 & -8.76 & rectum & 44.18 & -8.24 & -0.68 \\
cerebellum & -18.23 & 2.80 & -7.04 & salivary gland & 7.07 & -0.86 & 2.80 \\
cerebral cortex & -26.04 & 3.50 & -8.07 & seminal vesicle & 12.69 & -6.58 & -5.35 \\
cervix, uterine & -5.66 & 1.05 & 17.42 & skeletal muscle & -17.01 & 16.78 & -8.68 \\
colon & 5.92 & 1.88 & -2.34 & skin 1 & -17.85 & 5.41 & 11.50 \\
duodenum & 46.06 & -9.02 & -11.13 & skin 2 & 2.52 & 1.13 & 30.98 \\
endometrium 1 & -15.61 & -5.47 & -2.09 & small intestine & 45.14 & -8.14 & -11.36 \\
endometrium 2 & -18.97 & -3.34 & -1.29 & smooth muscle & -26.97 & 13.78 & -3.71 \\
epididymis & 17.71 & -4.04 & 0.13 & soft tissue 1 & -33.03 & 9.53 & -4.04 \\
esophagus & 9.24 & -1.28 & 30.81 & soft tissue 2 & -34.74 & 9.87 & -4.15 \\
fallopian tube & 17.88 & -6.94 & 4.60 & spleen & -29.78 & -2.72 & -1.35 \\
gallbladder & 44.89 & -2.42 & -6.33 & stomach 1 & 38.90 & -6.09 & -9.37 \\
heart muscle & -5.24 & 10.98 & -17.98 & stomach 2 & 37.32 & -8.03 & -8.16 \\
hippocampus & -27.72 & 2.91 & -9.28 & testis & -2.01 & 0.61 & -6.83 \\
kidney & 3.37 & 0.40 & -6.97 & thyroid gland & 17.91 & 2.35 & -1.42 \\
liver & -25.80 & -1.76 & -15.74 & tonsil & -6.95 & -7.24 & 10.08 \\
lung & -13.82 & 4.32 & -3.96 & urinary bladder & 24.77 & -7.25 & 10.34 \\
lymph node & -18.88 & -6.54 & 4.46 & vagina & -8.60 & 4.66 & 31.52 \\
nasopharynx & 23.74 & 0.54 & 10.79 & & & & \\
\hline
\end{tabular}


Suppl Table IV: Coordinates along the first three PC axes of the PE values for the 20 tumors from the HPA portal. By comparing with the corresponding normal tissues one may notice that, in this case, the transition from normal to tumor involves mainly a displacement along the $\mathrm{PC} 2$ axis.

\begin{tabular}{llll}
\hline Cancer type & PC1 & PC2 & PC3 \\
\hline breast cancer & -4.53 & -24.75 & -0.16 \\
carcinoid & -12.87 & -19.66 & -7.08 \\
cervical cancer & -16.05 & -22.45 & 8.47 \\
colorectal cancer & 7.57 & -31.04 & -0.22 \\
endometrial cancer & -9.84 & -26.58 & -3.70 \\
glioma & -23.86 & -11.22 & -1.82 \\
head and neck cancer & -8.83 & -22.27 & 10.95 \\
liver cancer & -9.46 & -20.67 & -10.79 \\
lung cancer & -18.29 & -22.12 & 2.25 \\
lymphoma & -28.17 & -15.76 & 1.09 \\
melanoma & -11.09 & -20.83 & 0.05 \\
ovarian cancer & -6.26 & -23.82 & 1.03 \\
pancreatic cancer & -4.42 & -24.35 & -1.46 \\
prostate cancer & -7.77 & -24.14 & -8.31 \\
renal cancer & -27.32 & -13.29 & -9.09 \\
skin cancer & -22.78 & -15.93 & 13.61 \\
stomach cancer & -9.80 & -24.99 & -0.96 \\
testis cancer & -13.20 & -22.50 & 3.99 \\
thyroid cancer & 3.31 & -23.28 & -7.05 \\
urothelial cancer & -10.58 & -22.48 & 6.51 \\
\hline
\end{tabular}

